\title{
ANTIFERROMAGNETISM IN REDUCED DIMENSIONAL SEMICONDUCTOR STRUCTURES
}

\author{
F. Schinagl, H. Krenn \\ Institut für Halbleiterphysik, Johannes Kepler Universität Linz \\ 4040 Linz, Austria, e-mail: f.schinagl@hlphys.uni-linz.ac.at
}

(Received March 8, 1998)

\begin{abstract}
We investigate layered nanostructures of the type II antiferromagnetic semiconductors separated by diamagnetic spacers. The magnetic ordering is described by isotropic Heisenberg exchange and dipolar interaction including an external magnetic field. According to the Hohenberg-MerminWagner theorem, two-dimensional short ranged Heisenberg systems do not order at all. This does hold no longer as soon as dipolar interactions or other anisotropies are considered. Mean field predictions for the Néel Temperature $T_{N}$ of EuTe/PbTe heterostructures are in good agreement with the measured values.
\end{abstract}

Key words: magnetic multilayers, phase transitions, magnetic semiconductors.

PACS number(s): 05.70.Fh, 75.50.Pp, 75.70.Ak

Among the low dimensional magnetic/non-magnetic superlattice structures antiferromagnetic insulators are ideal candidates for the study of phase diagrams and of dimensional crossover phenomena in thin films. The main reasons are that in contrast to the itinerant metallic layered systems the magnetic interactions in antiferromagnetic insulators are of short range and well localized. Most investigations in literature $[1,2]$ concern the antiferromagnets $\mathrm{NiO}, \mathrm{CoO}, \mathrm{CoF}_{2}, \mathrm{FeF}_{2}$ which can be grown on lattice matched diamagnetic layers of $\mathrm{ZnF}_{2}, \mathrm{MgF}_{2}$ or on amorphous layers like $\mathrm{SiO}_{2}$. These insulating superlattices exhibit ordering temperatures near room temperature and belong to the Ising type universality class of dimension two due to their strong magnetic anisotropy along the growth direction. These artificial structures show a magnetic behaviour different from their bulk properties because in reduced dimensions additional interactions appear to be dominant [3]: biquadratic besides bilinear exchange interaction, fourth order anisotropy terms and modified dipolar interactions. Also finite size effects $[4,5]$ and new magnetic phases beyond the standard three dimensional antiferromagnetic spin-flop and paramagnetic phases can be observed, e.g. asymmetric and spiral phases dependent on the thickness of the magnetic layers.

In our study we investigate low dimensional antiferromagnetic semiconductor (111) EuTe with a strong out of plane anisotropy ( $x y$ character). We start from a bilinear isotropic Heisenberg Hamiltonian and include dipolar interactions. We calculate the dependence of the Néel temperature on the reduction of the layer thickness using mean field approximation and we obtain special phases (parallel, spin-flop, antiparallel) with respect to an external applied magnetic field. The model is able to derive the whole phase diagram in the mean field picture.

The antiferromagnetism of EuTe is of type II, alternating ferromagnetic (111) layers form a staggered magnetisation. The exchange interactions are short ranged with twelve ferromagnetic nearest neighbours $\left(J_{\mathrm{NN}}>\right.$
$0)$ and six antiferromagnetic next nearest neighbours $\left(J_{\mathrm{NNN}}<0\right)$. These couplings are distributed within the (111) plane and across the neighbouring planes. Therefore for reduced dimensional EuTe some of these interactions are truncated and modify the magnetic structure. From neutron studies it is well known that the Eu spins are oriented within (111) planes having a very low in-plane anisotropy. Dipolar interaction is responsible for the easy plane-like behaviour. For thin layers only a single (111) domain remains out of the four equivalent domains which exist in bulk material. The growth plane coincides with the magnetic easy plane. Alternating spin layers are stacked on each other. The Eu spin originates from a halve filled $4 \mathrm{f}$ shell with configuration $[\mathrm{Xe}] 4 \mathrm{f}^{7} 5 \mathrm{~d}^{0} 6 \mathrm{~s}^{2}$ and is well localized. It is treated as a classical spin vector with $s=\frac{7}{2}$. The nature of exchange interactions $J_{\mathrm{NN}}, J_{\mathrm{NNN}}$ can be described in terms of band mediated and super exchange interactions [6,7]. If one includes the dipolar interaction the isotropic Heisenberg Hamiltonian is modified.

The Hamiltonian in a homogeneous external magnetic field $\mathbf{H}$ reads

$$
\mathcal{H}=-\sum_{l>l^{\prime}} \sum_{\alpha \beta}\left(J_{l l^{\prime}} \delta^{\alpha \beta}+A_{l l^{\prime}}^{\alpha \beta}\right) S_{l}^{\alpha} S_{l^{\prime}}^{\beta}-g \mu_{\mathrm{B}} \mathbf{H} \sum_{l} \mathbf{S}_{l}
$$

with spins $\mathbf{S}_{l}$ at lattice sites $\mathbf{x}_{l}$. The first term in brackets is the exchange interaction $J_{l l^{\prime}}$ ( $J_{\mathrm{NN}}$ for nearest neighbours, $J_{\mathrm{NNN}}$ for next nearest neighbours and zero otherwise). The second term is the usual dipole-dipole interaction

$$
A_{l l^{\prime}}^{\alpha \beta}=-\frac{\mu_{0}}{4 \pi} g^{2} \mu_{\mathrm{B}}^{2}\left(\frac{\delta^{\alpha \beta}}{\left|\mathbf{x}_{l}-\mathbf{x}_{l^{\prime}}\right|^{3}}-3 \frac{x_{l}^{\alpha} x_{l^{\prime}}^{\beta}}{\left|\mathbf{x}_{l}-\mathbf{x}_{l^{\prime}}\right|^{5}}\right)
$$

$\alpha, \beta$ denote the Cartesian components of the vectors $\mathbf{x}$ and $\mathbf{S}$. The second term in this equation introduces an 
anisotropy in the otherwise isotropic Hamiltonian.

We define a set of mean field equations according to the thermally averaged spins at location $l^{\prime}$

$$
\left\langle\mathbf{S}_{l^{\prime}}\right\rangle=\frac{\mathbf{X}_{l^{\prime}}}{\left|\mathbf{X}_{l^{\prime}}\right|} s \mathrm{~B}_{s}\left(\frac{g \mu_{\mathrm{B}} s\left|\mathbf{X}_{l^{\prime}}\right|}{k T}\right)
$$

$\mathbf{X}_{l^{\prime}}$ is the effective molecular field at site $l^{\prime}$

$$
X_{l^{\prime}}^{\beta}=H^{\beta}+\frac{2}{g \mu_{\mathrm{B}}} \sum_{l \neq l^{\prime}} \sum_{\alpha}\left(J_{l l^{\prime}} \delta_{\alpha \beta}+A_{l l^{\prime}}^{\alpha \beta}\right)\left\langle S_{l}^{\alpha}\right\rangle
$$

and $\mathrm{B}_{s}(x)$ is the Brillouin-function for spin $s$.

The mean field Hamiltonian $\mathcal{H}^{\prime}$ neglects fluctuations and replaces the spins by their thermal averages and the magnetic field by the effective field:

$$
\begin{aligned}
\mathcal{H}^{\prime} & =\sum_{l \neq l^{\prime}} \sum_{\alpha \beta}\left(J_{l^{\prime}} \delta_{\alpha \beta}+A_{l^{\prime}}^{\alpha \beta}\right)\left\langle S_{l}^{\alpha}\right\rangle\left\langle S_{l^{\prime}}^{\beta}\right\rangle \\
& -g \mu_{\mathrm{B}} \sum_{l} \sum_{\alpha} X_{l}^{\alpha} S_{l}^{\alpha} .
\end{aligned}
$$

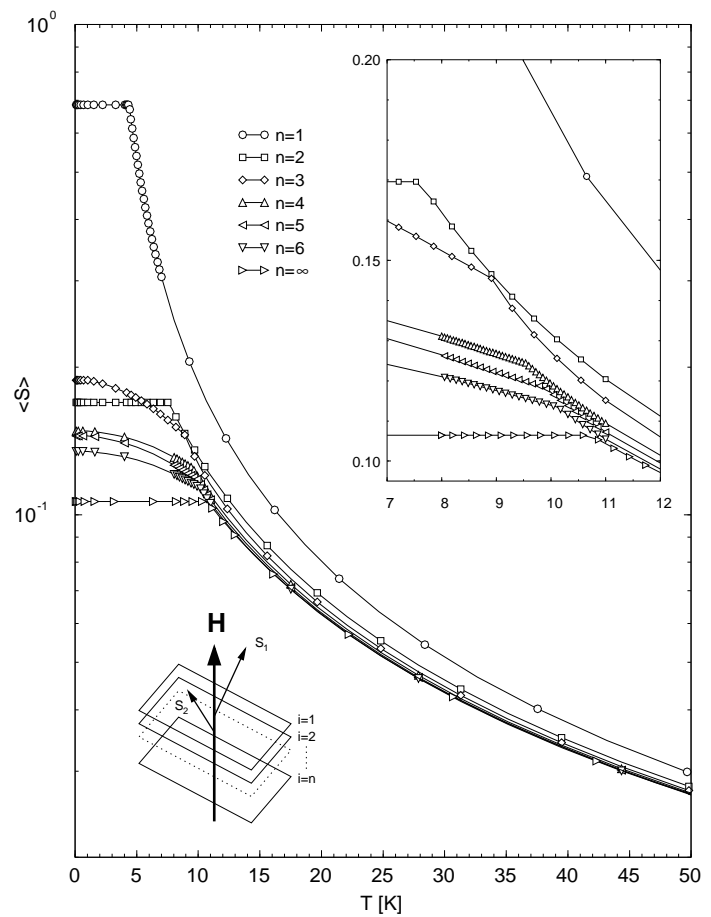

(a) $\mathbf{H}$ perpendicular to the planes.

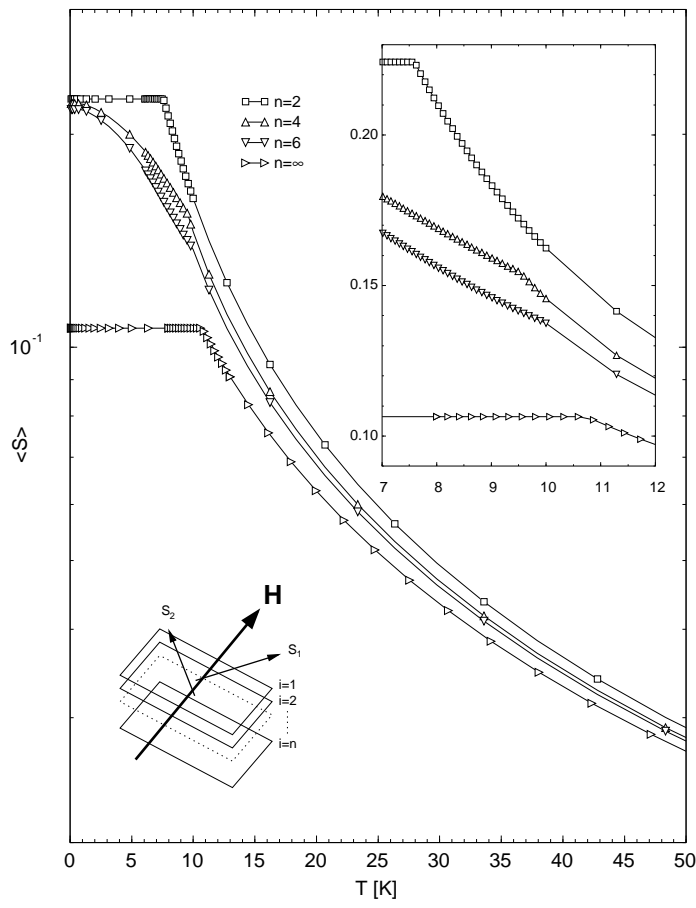

(b) $\mathbf{H}$ in-plane, flopped spins for even $\mathrm{n}$ only.

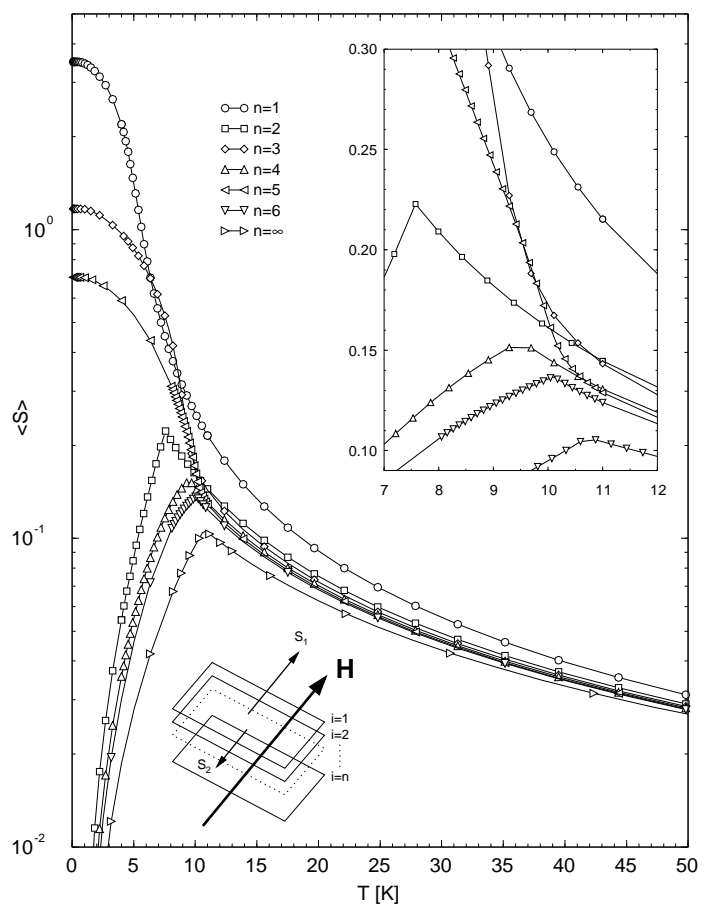

(c) $\mathbf{H}$ in-plane with collinear spin orientation.

Fig. 1. Average spin per Eu atom of $n=1$ up to $n=6$ EuTe monolayers as a function of temperature $T$ in an external magnetic field $H=2 \mathrm{kOe}$. The insets show magnified views of the region near the phase transition temperature $T_{N}(n) . n=\infty$ corresponds to three dimensional (bulk) EuTe. 


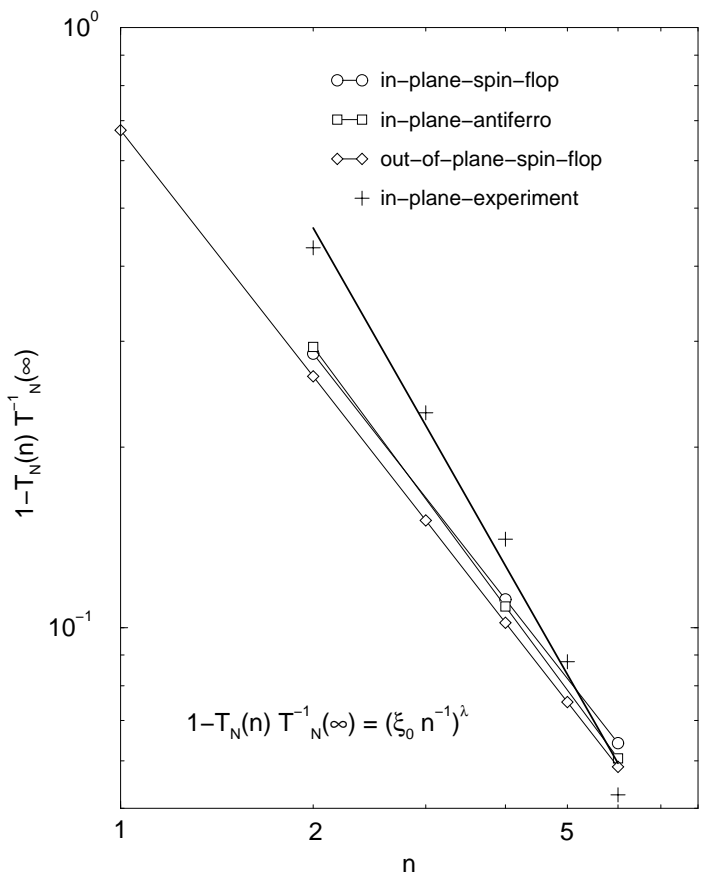

Fig. 2. Normalized shift of the phase transition temperature $T_{N}(n)$ versus number $n$ of monolayers EuTe in an external magnetic field $H=2 \mathrm{kOe}$. Crosses and bold line denote experimental (exp) data. Circles, squares and diamonds show results of mean field ( $\mathrm{mf}$ ) calculations. The values of the shift exponent $\lambda$ and correlation length critical amplitude $\xi_{0}$ are given in the text. $T_{N}(\infty)$ is the Néel temperature of bulk EuTe: $T_{N}^{\exp }(\infty)=10.08 \mathrm{~K}$ and $T_{N}^{\mathrm{mf}}(\infty)=10.69 \mathrm{~K}$.

The mean field equations are solved selfconsistently for the $\left\langle S_{l}^{\alpha}\right\rangle$ which means that at each site $l$ the effective field $\mathbf{X}_{l}$ lines up parallel with $\left\langle\mathbf{S}_{l}\right\rangle$. The corresponding mean field Hamiltonian gives the best possible approximation to the true free energy of the system [8]. To reduce the infinite set of mean field equations we restrict the system to a finite-sized hexagonal cluster with periodic in-plane (111) boundary conditions with a perpendicular extension of a stack of $n$ planes aligned according to the fcc structure along the [111] direction. In order to rule out incommensurate spin arrangements the size of the cluster has been varied from three up to seven spins per edge of the hexagon. Within this range we find identical solutions. The calculations yield the staggered spin arrangements along the [111] direction with ferromagnetically organized (111) planes. One should recall that the $x y$ character of spin alignment is caused by the dipolar interaction.

We investigate EuTe layers with the thickness ranging from $n=1$ up to $n=6$ monolayers and in addition $n=\infty$ by using a cubic cluster with periodic boundary conditions along all three spatial extensions. For $n=\infty$ (bulk case) we compared the experimentally observed phase transition temperature $\left(T_{N} \simeq 10.08 \mathrm{~K}\right)$ with the calculated one ( $T_{N} \simeq 10.69 \mathrm{~K}$ ) by choosing the exchange parameters $J_{1}=0.06 \mathrm{~K}$ and $J_{2}=-0.16 \mathrm{~K}$ from the liter- ature [9]. We adopt the same values for the investigation of the reduced dimensional systems.

In accordance with experiments we align the external magnetic field $\mathbf{H}$ to a perpendicular or in plane direction. In the latter case it can be shown that due to a lack of any in-plane anisotropy all magnetic field directions are equivalent within the ferromagnetic sheets. The model explicitly yields two in-plane solutions: the spinflop state and the antiferromagnetic state. It comes out that the local order parameter $\left\langle\mathbf{S}_{l}\right\rangle$ depends only on the location of the sublayer: the spin orientation within an individual (111) layer is independent of its location in the plane. In Fig. 1 we present the spatially averaged order parameter $\langle S\rangle$ as a function of temperature for a magnetic field $H=2 \mathrm{kOe}$ perpendicular to the planes (Fig. 1a), the spin-flop in-plane magnetic field (Fig. 1b) and the in-plane field aligned with a sublayer magnetisation (Fig. 1c). In Fig. 1a a single ferromagnetic EuTe monolayer shows the maximum magnetisation. The constant magnetisation below $T_{c} \simeq 4.8 \mathrm{~K}$ arises from spins which are canted out of plane. Its magnitude depends only on the strength of dipolar coupling. In contrast, in the absence of dipolar interaction one would expect the saturation values $\langle S\rangle=s$. For $n>1$ the exchange interaction competes with the Zeeman and dipolar interaction and yields a reduced magnetisation. For $n>2$ the sublattice magnetisations are generally not compensated which gives a partial increase of the magnetisation. For bilayers and bulk like systems perfect compensation is observed.

For a magnetic field $\mathbf{H}$ in plane (Fig. 1b) a similar behaviour for the so called spin-flop phase is expected. Systems with odd numbers of monolayers $n$ are unstable in the spin-flop phase. Therefore in Fig. 1b only the compensated even numbered systems are shown. They exhibit a plateau or ferrimagnetic like behaviour, respectively, below the phase transition temperature $T_{N}$ which is higher susceptible in magnetic field than the out of plane field direction. The spin-flop phase is energetically favoured with respect to the antiferromagnetic phase Fig. 1c.

In the collinear antiferromagnetic phase the sublattice magnetisations are aligned with the in-plane field. As expected from the common antiferromagnetic behaviour the magnetisation increases for increasing temperature as long as $T<T_{N}$. It is obvious that odd numbers of monolayers contribute to an uncompensated (ferri-) magnetic moment.

For all three phases according to Figs. $1 \mathrm{a}-\mathrm{c}$ the phase transition temperature is plotted in Fig. 2 as a function of the number of monolayers (ML) $n$ together with experimental data. We fitted the calculated and measured phase transition temperatures to the finite-size scaling relation [4]

$$
\frac{T_{N}(\infty)-T_{N}(n)}{T_{N}(\infty)}=\left(\frac{\xi_{0}}{n}\right)^{\lambda}
$$

The shift exponent $\lambda$ is related to the critical exponent $\nu$ 
of the bulk $(n \rightarrow \infty)$ correlation length: $\lambda=\nu^{-1}$. In our case of a continuous phase transition, finite size scaling is important if the correlation length $\xi=\xi_{0}\left|\frac{T-T_{N}(n)}{T_{N}(\infty)}\right|$ exceeds the thickness of the film. The relations Eq. (3) and $\lambda=\nu^{-1}$ are based on the validity of the hyperscaling relation $d \nu=\gamma+2 \beta$ (with $d$ the dimension of the system and $\gamma$ and $\beta$ the critical exponents of susceptibility and the magnetisation, respectively) and on the occurrence of a unique single correlation length. Even for $d>4$ correct finite size scaling cannot be deduced from mean field theory as it is demonstrated for standard $\phi^{4}$ field theory [10].

From our calculations Eqs. (1), (2) we find $\nu=0.72$ which deviates from $\nu=0.5$ as anticipated by mean field theory. In contrast, from measured data we extract $\nu_{\text {meas }}=0.53$ quite close to the mean field value and a critical amplitude $\xi_{0}^{\text {meas }}=1.3 \mathrm{ML}$. This discrepancy cannot be clarified at the moment but we conjecture it originates from the long range character of dipolar interaction.

Summarizing, the temperature dependent magnetisation behaves nonmonotonically for even $n$ whereas for odd $n$ magnetisation decreases smoothly $(H>0)$ with increasing temperature (Fig. 1c). In the latter case the phase transition is quenched like for ferromagnets in a finite magnetic field. For the compensated antiferromagnetic-like systems (even $n$ ) the magnetic field is an irrelevant variable for the order parameter and does not influence the antiferromagnetic phase transition.

As has been proved by N. D. Mermin and H. Wagner [11] one or two dimensional isotropic spin-s Heisenberg models with finite range exchange interaction can be neither ferromagnetic nor antiferromagnetic at any nonzero temperature. This conclusion cannot be reproduced by mean field analysis. However if one includes dipolar interaction C. Pich and F. Schwabl [12] have shown that long range order is possible in two dimensional Heisenberg antiferromagnets. In this context mean field analysis becomes tractable to explain the experimentally observed phase transitions in antiferromagnetic 2D layers. In addition dipolar interaction can mediate long range interlayer couplings across non-magnetic spacer layers like in superlattice samples. Such systems can induce a rich variety of phase transition phenomena.

Support by the "Austrian Fonds zur Förderung der Wissenschaftlichen Forschung", grant 11557, is acknowledged.
[1] E. N. Abarra, K. Takano, F. Hellman and A. E. Berkowitz, Phys. Rev. Lett. 77, 3451 (1996).

[2] C. A. Ramos et al, Phys. Rev. Lett. 65, 2913 (1990).

[3] M. L. Plumer, A. Caillé and K. Hood, Phys. Rev. B 39, 4489 (1989).

[4] M. E. Fisher and M. E. Barber, Phys. Rev. Lett. 28, $1516(1972)$.

[5] D. Lederman, C. A. Ramos, V. Jaccarino and J. L. Cardy, Phys. Rev. B 48, 8365 (1993).

[6] Robert H. Swendsen, Phys. Rev. B 5, 116 (1972).
[7] Robert H. Swendsen, Phys. Rev. B 11, 1935 (1975).

[8] J. M. Yeomans, Statistical Mechanics of Phase Transitions (Clarendon Press, Oxford, 1992).

[9] W. Zinn, J. Magn. Magn. Mater. 3, 23 (1976).

[10] X. S. Chen and V. Dohm, submitted to Phys. Rev. Lett. (1997).

[11] N. D. Mermin and H. Wagner., Phys. Rev. Lett. 17, 1133 (1966).

[12] C. Pich and F. Schwabl, Phys. Rev. B 47, 7957 (1993). 